\title{
Would Recessionary Shock Induce More Intermediation in the Informal Sector?
}

\author{
Biswajit Mandal \\ Visva-Bharati University
}

\begin{abstract}
This paper attempts to give an idea about the upshot of a recessionary phase on the intermediation activity which is required for the survival of the informal fragment of a society. Informal part of the economy covers a large chunk of the total economic activities in any developing economy. Therefore it is imperative to check the possible consequences of recession on this subdivision because intermediation also requires unskilled work force which need to be pulled out from the productive spectrum. In doing so I have used the hybrid of standard Heckscher-Ohlin and Specific Factor general equilibrium models of trade. In such a framework it has been shown that a recessionary shock may induce an expansion in intermediation activity under certain factor intensity assumption.
\end{abstract}

- JEL Classification: F11, D73, O17, D5.

- Keywords: International Trade, Corruption, Informal sector, General Equilibrium

\section{Introduction}

The existence of informal segment in an economy is not an abnormality now-adays. Irrespective of the development status of any country, informal segment occupies a sizeable amount of total employment. The informal sector covers primarily the non-agricultural employment of unskilled labor. It accounts for 5080\% employment in South Asia, 30-50\% in South East Asia, 40-50\% in Africa, 55\% in Latin America and Caribbean, 24\% in Southern Europe, 10\% in Western

\footnotetext{
*Corresponding address: Biswajit Mandal; Department of Economics \& Politics, Visva-Bharati University, Santiniketan, India, 731235. Telephone: (+91) 03463262751-56 Extn. 405, E-mail: biswajiteco@gmail.com. biswajit.mandal@visva-bharati.ac.in @2011-Center for Economic Integration, Sejong Institution, Sejong University, All Rights Reserved.
} 
Europe, $18 \%$ in Canada and $8 \%$ in USA (Dijkstra, 2006).

In the post recession phase all segments of the economy have suffered to some extent. Informal sector is no exception. A few papers have already been written to capture the post-recession reality of the developing nations that comprises of both formal and informal zones. Chaudhuri (2009) and Marjit et al (2011) have nicely brought into the concept of informal sector in a standard theoretical model and explained some likely consequences. Chaudhuri (2009), using a Harris-Todaro kind of framework has explained how recession could put a mark on the informal workers. Marjit et al (2010) included a tradeable informal good in a general equilibrium model to check the robustness of an increase in informal wage due to recession.

However, till date a few attempts have been made in the literature to capture another reality that informal sector is always beset with extortion or corruption as the survival of the so-called extra-legal activity crucially depends on the negotiation between informal producers and administrators. In an interesting paper Friedman, Johnson, Kaufman and Zoido-Lobaton (2000) have found the existence of corruption and production both as separate activities within the informal sector. One may easily argue over the type of corruption, nature of negotiation required in this fragment but the undeniable fact is that corruption related intermediation is omnipresent in the informal counterpart of the society. ${ }^{1}$ This sort of intermediation or manipulation is precisely done by a group of labor who are termed as intermediators or extortionists. Intermediators are paid out of the price of informal good(s). Therefore this paper attempts to look at the possible effects of a recessionary shock on the informal unproductive or corruption related intermediation fragment of an economy. Thus I shall try to invoke this very phenomenon in a standard trade theoretic general equilibrium set up. Three earlier papers by Marjit and Mandal (2008), Mandal and Marjit (2010) and Mandal and Chaudhuri (2011) have used the similar notion to consider such an issue of corruption and/or intermediation. The current paper uses the Heckscher-Ohlin nugget kind of framework developed in Gruen and Corden (1970), Jones and Marjit $(1992,2009)$ that resembles complementarity structure. This is essentially a hybrid of Heckscher-ohlin and Specific factor models of trade. This structure has more often

\footnotetext{
${ }^{1}$ For a detailed analysis of the causes of the existence of informal sector interested readers may look at Marjit and Kar (2009), Marjit (2009). Marjit and Kar (2009) clearly elaborates the interconnectedness of the informal sector with other sectors of the economy and some policy implications.
} 
than not been used to assess the development policies.

The paper is schematized as follows. Introduction is followed by the model in section II. Section III delivers the basic result with explanations. Section IV contains concluding remarks.

\section{The Model}

The economy that I consider here is a small one characterized by the coexistence of formal and informal subdivisions. $X$ and $Y$ are traded and are being produced in the formal set up following neo-classical production function. $X$ uses skilled labor and capital whereas $Y$ uses unskilled workforce and capital. Capital is perfectly mobile across sectors and goods. Note that unskilled workers' wage is pre-determined by trade union in $Y$. There is another commodity, $Z$, the informal good. $Z$ is being produced by the non-unionized unskilled workers and capital. Unskilled wage in the informal sector is expectedly less than unionized wage since who do not find jobs in the formal part fall back on the informal counter part where wage can have a free fall following competitive mechanism. No one can afford to remain unemployed. This is precisely the reason why informal sector exists. The existence of informal sector also ensures the full employment criterion for unskilled labor.

Corruption related intermediation in our framework switches only unskilled labor from productive to corruptive activities. Thus corruption is viewed as directly unproductive profit seeking activity (Bhagwati, 1982) as some people engaged in corruption essentially avail of the arbitrage opportunities, acting as middlemen and intermediaries. Such diversion of human talent can be quite costly for the society as effective labor endowment shrinks. $\alpha$ is the fraction of value of per unit $Z$ that could be spent on extortionists for doing intermediation allied assignment. This is done only by unskilled workers. Intermediation/extortion sector is represented by $C$. Thus unskilled workers have three options: to work in $Y$ or in $Z$ or in $C$. All the markets are competitive. Competitive market for extortion ensures that lost value of output would be exactly equal to the payment made to the unskilled workers who actually do the intermediations.

The system of equations would be as follows. Note that we would be intimately following the structure of Marjit and Mandal (2008) and be using the standard symbols that are frequently used in stylized general equilibrium trade models (Jones, 1965; Jones 1971). Here, $P_{j} \Rightarrow$ price of the $\mathrm{j}^{\text {th }}$ commodity $(j=X, Y, Z$ ); 
$w_{s} \Rightarrow$ skilled wage; $\bar{w} \Rightarrow$ unskilled formal wage; $w \Rightarrow$ unskilled informal wage; $r \Rightarrow$ rate of return to $K ; \alpha_{i j} \Rightarrow$ share of the $\mathrm{i}^{\text {th }}$ factor in $\mathrm{j}^{\text {th }}$ commodity ( $i=S, L, K$ and $j=X, Y, Z) ; S \Rightarrow$ total supply of skilled labor; $\bar{L} \Rightarrow$ total supply of unskilled labor; $L_{C} \Rightarrow$ number of unskilled labor employed in extortion; $\bar{K} \Rightarrow$ total supply of capital, $K$.

Non-existence of supernormal profits entails that the competitive price equations be:

$$
\begin{gathered}
w_{s} \alpha_{s x}+r a_{k x}=P_{x} \\
\bar{w} a_{l y}+r a_{k y}=P_{y} \\
w a_{l z}+r a_{k z}=P_{z}(1-\alpha)
\end{gathered}
$$

Note that $\bar{w}>w$ because of the trade union exercise in the formal unskilled segment.

$$
w \cdot L_{C}=\alpha \cdot P_{z} \cdot Z
$$

Factors' full employment conditions are defined as:

$$
\begin{gathered}
a_{s x} \cdot X=\bar{S} \\
a_{l y} \cdot Y+a_{l z} \cdot Z=\bar{L}-L_{c} \\
a_{k x} \cdot X+a_{k y} \cdot Y+a_{k z} \cdot Z=\bar{K}
\end{gathered}
$$

The structure we just built is merely a recursive one. $X$ uses a specific factor in form of skilled labor $(S)$. $Y$ and $Z$ share the unskilled labor as the mobile factor $(L)$. Whereas capital $(K)$ is perfectly mobile across all $X, Y$ and $Z$. It is important to keep in mind throughout that $\alpha$, the degree of corruption or extortion is exogenous in the model.

We have some typical essential phenomena in our model. All goods' prices are determined from the international market and the formal unskilled workers get fixed wage rate. Formal unskilled wage is, essentially, the prime driving force of this paper. The informal good's price is considered to be constant (without losing generality, an introduction of a Cobb-Douglas type of preference function for $Z$ would not qualitatively change the essence of the structure). Nonetheless we can 
easily induce some changes in $P_{z}$. Subsequently there will be further strengthening or a bit dampening effect on $w$. This will again, of course, persuade some further changes in output and intermediation front. Therefore, once the recessionary phase starts, by virtue of the structure of the model, capital gets the first jostle as unskilled wage is pre-fixed in the formal segment. Capitalists, internalizing the distress, would help increasing the skilled workers' wage if the price of skilled goods does not alter. However, as the informal good's price remains unchanged, the informal workers must gain as the return to the mobile factor has already gone down. However, in output front there might be some interesting outcomes. After being disappointed in the formal unskilled segment, capital immediately flows out and subsequently the output contracts. Whereas, in the skilled segment, producers try to substitute skilled labor by relatively less costly capital. Thus the output effect here depends on the relative changes in factor return. Nevertheless, in the informal sector, as wage goes up, there could be some changes in $L_{C}$. The eventual output effects on $Y$ and $Z$ will substantially depend on the factor intensity comparison between $Y$ and $Z$. Needless to say that in this paper my prime focus would be on $L_{C}$ or $w . L_{C}$, the size of the intermediation activity.

Here equation (1) represents the specific factor structure while equations (2) and (3) resemble the Heckscher-Ohlin subsystem. This is how the hybrid nature of our model comes into play. $P_{x}$ and $P_{y}$ are determined in the world market. $r$ is solved from equation (2) as wage is pre-negotiated at $\bar{w} \cdot w_{s}$ can be easily calculated from (1) since $r$ is already determined. Thus non-unionized informal wage, $w$ is determined from (3) for any given $\alpha$ and $P_{z}$. By means of CRS (constant Returns to Scale) and DMP (Diminishing Marginal Productivity of factors) assumptions all $\alpha_{i j}$ s are determined. Now let us start from any positive $L_{C}$ such that $\left(\left(\bar{L}-L_{C}\right)>0\right)$. Hence $\mathrm{X}$ is derived from (5) and $\mathrm{Y}$ and $\mathrm{Z}$ are simultaneously determined from (6) and (7) for given factor endowments.

Moreover, we can also solve for the equilibrium value of $L_{C}$ or the size of the extortion sector in our model. Once $w$ is determined the LHS (Left Hand Side) of equation (4) would be an increasing function of $L_{C}$ with slope $w$. As $L_{C}$ goes up, availability of productive unskilled labor shrinks. Thus what would happen to the RHS (Right Hand Side) of (4) and output of $Z$ that crucially depends on the factor intensity assumption of $Z$ compared to $Y$. If $Z$ happens to be the labor-intensive one, output of $Z$ must fall. Rationality suggests the assumption of a labor-intensive $Z$. Therefore the RHS of (4) must be declining in $L_{C}$ for given given $\alpha$ and $P_{z}$. Hence the value of equilibrium $L_{C}$ is worked out for given degree of extortion, w 
Figure 1. Determination of equilibrium $L_{C}$ for given $w, P_{Z}$ and $\alpha$

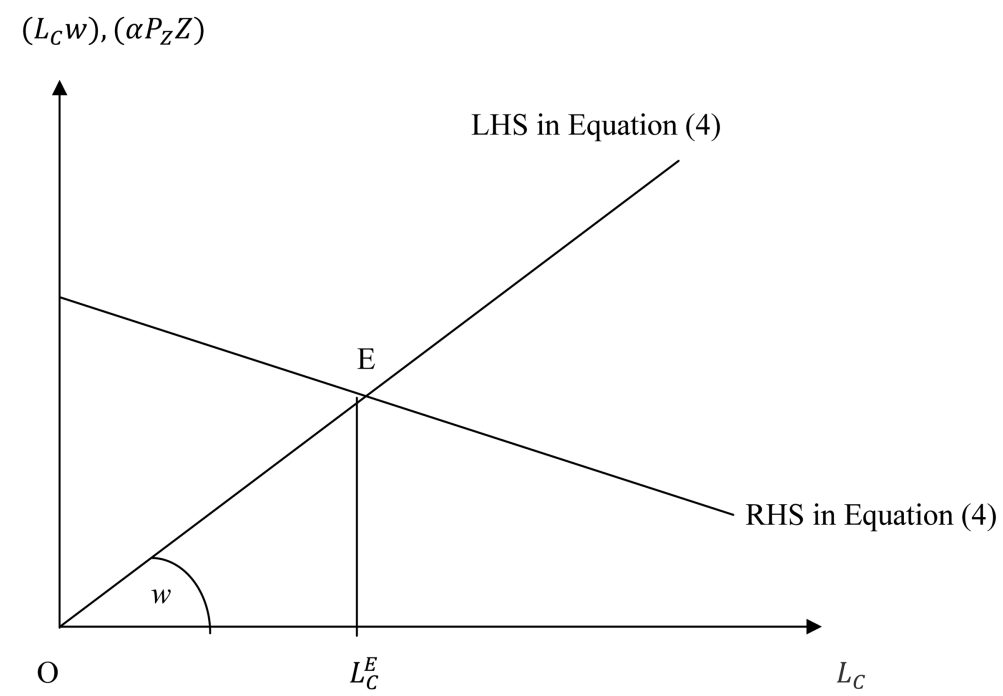

and $P_{z}$. Determination of equilibrium $L_{C}$ is shown in diagram-1.

In this model, in conjunction with price effect caused by recessionary shock, the real possibility of endowment effect is explored. This is not the kind of Rybczynski effect which originates from a change in factor return (making the constraint less or more binding). In this model I have, in fact, two types of Rybczynski effects. One originates from the change in factors' return, the conventional way to look at the complementarity structure. The other one comes from the direct change in the unskilled labor endowment for productive purpose. Once the return to unskilled workers changes, the output of $Y$ and $Z$ will change, depending upon the intensity of factor used. With the change in $Z$ the number of intermediators is likely to vary. This will again initiate another round of Rybczynski effect. Therefore the second category of Rybczynski effect essentially enters through the change in the size of the intermediation sector that requires only unskilled labor which has another alternative employment options in the production of $Y$ and $Z$.

\section{Basic Results}

As I have mentioned before, in this paper my prime focus would be to check the potential effects on the size of the intermediation or extortion sector consequent upon recessionary shocks. For this purpose let me start from some facts that are 
explored in UNCTAD secretariat calculations (2009). The report emphasizes that there is $37 \%$ decline in the price of all commodities' group excluding crude petroleum in between 2008 and January 2009. The crude petroleum price in isolation has been decreased by $67 \%$ in the same period. In case of service trade it has been observed that service export growth had started declining in the third quarter of 2008 with a sudden fall in the last quarter of 2008. Therefore, it is, by and large, accepted that prices of traded goods have been reduced in the phase of economic recession. ${ }^{2}$

The comparative static properties of this model can be established by considering the effects of changes in the parameters like goods' prices. In this model the resultant effect of a decrease in the prices of the formal goods would be first felt in $Y$. As wage is pre-fixed, the first shove would be on the capital and thus $r$ would fall following a decrease in $P_{y}$. Now, what is going to happen to that depends on the relative strengths of a fall in $r$ and $P_{x}$. Nevertheless, $w$ would inevitably increase in the informal sector as $\alpha$ and $P_{Z}$ are assumed to be fixed for the time being. The change in $w$ will invoke some adjustment in the size of the intermediation sector and subsequent changes in the commodity output. This would again lead to another round of adjustment in equation (4). Now, let me try to figure out the mathematical channels through which this model works.

Totally differentiating the price equations we have

2

Table 1. The steep fall in traded commodity prices

\begin{tabular}{lccc}
\hline Commodity group & Peak in 2008* & Jan 2009* & \% change \\
\hline All commodities (excluding crude petroleum) & 299.5 & 189.4 & -37 \\
Food & 280.6 & 196.5 & -30 \\
Tropical beverages & 193.5 & 165.5 & -15 \\
Vegetable oilseeds and oils & 370.5 & 191.7 & -48 \\
Agricultural raw materials & 228.6 & 145.6 & -36 \\
Minerals, ores and metals & 391.6 & 203.5 & -48 \\
Crude petroleum & 469.5 & 155.6 & -67 \\
\hline
\end{tabular}

Source: UNCTAD Secretariat calculations

* Price indexes of all and main commodity groups (in terms of current dollars). 


$$
\begin{gathered}
\hat{w_{s}} \theta_{s x}+\hat{r} \theta_{k x}=\hat{P}_{x} \\
\hat{\bar{w}} \theta_{l y}+\hat{r} \theta_{k y}=\hat{P}_{y} \\
\hat{w} \theta_{l z}+\hat{r} \theta_{k z}=\hat{P}_{z}(1-\alpha)-\mathrm{a} \hat{a}
\end{gathered}
$$

$\hat{\bar{w}}=0$ because $\bar{w}$ is always pre-determined.

Setting $\hat{\bar{w}}=0$ in equation (9) we can solve for $\hat{r}$ and then substituting it in (8) we get the value for $\hat{w}_{s}$. Hence

$$
\begin{gathered}
\hat{r}=\frac{\hat{P}_{y}}{\theta_{k y}} \\
\hat{w}_{s}=\left(\hat{P}_{x}-\frac{\hat{P}_{y}}{\theta_{k y}} \theta_{k x}\right) \frac{1}{\theta_{s x}}
\end{gathered}
$$

Again using (11) in (10) we can have the value of $\hat{w}$,

$$
\hat{w}=\left\{\hat{P}_{z}(1-\alpha)-\alpha, \hat{\alpha}-\hat{P}_{y} \frac{\theta_{k z}}{\theta_{k y}}\right\} \frac{1}{\theta_{l z}}
$$

The intuitive explanations for the value of changes are very simple to follow. When $P_{x}$ and $P_{y}$ fall,(assuming that $P_{z}$ remains constant ${ }^{3}$ and $\alpha$ remains constant throughout),

$$
\begin{aligned}
& \hat{r}<0 ; \\
& \hat{w}>04 \\
& \hat{w}_{s} \gtrless 0 \text { iff }\left|\frac{\theta_{k x}}{\theta_{k y}}\right| \gtrless\left|\frac{\hat{P}_{x}}{\hat{P}_{y}}\right|
\end{aligned}
$$

$\theta_{k x}$ is likely to be greater than $\theta_{k y}$ as skilled good normally requires more capital. Therefore, if $P_{x}$ falls less (more) than $P_{y}, w_{s}$ will increase (decrease).

\footnotetext{
${ }^{3}$ When the informal good is considered to be a substitute for the formal unskilled good price of the informal good should rise in the recessionary situation. Traditionally formal good's price is greater than that of informal good. People's demand for relatively cheaper good will increase and this would pull up $P_{z}$. ${ }^{4}$ If $P_{z}$ rises, $w$ will increase further.
} 
Differentiating equation (4) one gets,

$$
\hat{L_{C}}=\hat{\alpha}+\hat{P_{z}}+\hat{Z}-\hat{w}
$$

Implication of (14) is quite simple to understand. When $\alpha$ and $P_{z}$ are constant, an increase in $Z$ induces more people to be employed as intermediators while a simultaneous increase in $w$ would affect $L_{C}$ in opposite direction. Possibly, because, people's natural tendency is to work as productive factors not as an intermediators. Therefore, a positive $\hat{w}$ would lead to a fall in $L_{C}$.

Factors' return must change due to any change in the goods' price. However, as the endowments of factors are constant producer would go for substituting the factors in use depending on the elasticity of substitution, internal reallocation of factors etc. Hence there would be some changes in the production of $X, Y$ and $Z$.

Totally differentiating the full employment condition (5) - (7) and using the concept of elasticity of substitution one can have,

$$
\begin{gathered}
\hat{X}=\hat{\bar{S}}-\hat{\alpha_{s x}} \\
\hat{Y} \lambda_{l y}+\hat{Z} \lambda_{l z}=\lambda_{l z} \sigma_{z} \theta_{k z}(\hat{w}-\hat{r})-\lambda_{l y} \sigma_{y} \theta_{k y} \hat{r}-\hat{L_{C}} \lambda_{l c} \\
\hat{Y} \lambda_{k y}+\hat{Z} \lambda_{k z}=-\lambda_{k z} \sigma_{z} \theta_{l z}(\hat{w}-\hat{r})+\lambda_{k y} \sigma_{y} \theta_{l y} \hat{r}-\lambda_{k x} \sigma_{x}\left(\hat{w_{s}}-\hat{r}\right)
\end{gathered}
$$

Setting $\hat{\alpha}=\hat{P}_{z}=0$, equation (14) can be modified as

$$
\hat{L_{C}}-\hat{Z}=-\hat{w}
$$

Where, $\sigma_{x}=\frac{\hat{\alpha}_{k x}-\hat{\alpha}_{s x}}{\hat{w}_{s}-\hat{r}}, \sigma_{y}=\frac{\hat{\alpha}_{k y}-\hat{\alpha} l y}{\hat{\bar{w}}-\hat{r}}, \sigma_{z}=\frac{\hat{\alpha}_{k z}-\hat{\alpha}_{l z}}{\hat{w}-\hat{r}}$

Solving for $\hat{X}$ we get

$$
\hat{X}=\sigma_{x}\left(\hat{w}_{s}-\hat{r}\right) \theta_{k x}
$$

Depending on $\hat{w}_{s}, \hat{X}$ may take any value. However, the solutions for $\hat{Y}$ are $\hat{Z}$ not very straightforward. Because, the same unskilled labor is shared among $Y, Z$ and C. Thus, we need to go for simultaneous determination of $\hat{Y}, \hat{Z}$ and $\hat{L}_{C}$.

Equation (16), (17) and (18) can alternatively be represented in the following 
matrix form:

$$
\left(\begin{array}{ccc}
\lambda_{k y} & \lambda_{k z} & 0 \\
\lambda_{l y} & \lambda_{l z} & \lambda_{l c} \\
0 & -1 & 1
\end{array}\right)\left(\begin{array}{c}
\hat{Y} \\
\hat{Z} \\
\hat{L_{C}}
\end{array}\right)=\left(\begin{array}{c}
-\lambda_{k z} \sigma_{z} \theta_{l z}(\hat{w}-\hat{r})+\lambda_{k y} \sigma_{y} \theta_{l y} \hat{r}-\lambda_{k x} \sigma_{x}\left(\hat{w_{s}}-\hat{r}\right) \\
\lambda_{l z} \sigma_{z} \theta_{k z}(\hat{w}-\hat{r})-\lambda_{l y} \sigma_{y} \theta_{k y} \hat{r} \\
-\hat{w}
\end{array}\right)
$$

Using the Cramer's rule we can simultaneously solve for $\hat{Y}, \hat{Z}$ and $\hat{L_{C}}$.

$$
\begin{aligned}
& \hat{Y}=\frac{1}{|A|}\left[\lambda_{k y} \sigma_{y} \theta_{k y} \hat{r}-\lambda_{k x} \sigma_{x}\left(\hat{w}_{s}-\hat{r}\right)-\lambda_{k z} \sigma_{z} \theta_{l z}(\hat{w}-\hat{r})\left\{\lambda_{l z}+\lambda_{l c}\right\}\right. \\
& \left.-\lambda_{k z}\left\{\lambda_{l z} \sigma_{z} \theta_{k z}(\hat{w}-\hat{r})-\lambda_{l y} \sigma_{y} \theta_{k y} \hat{r}+\lambda_{l c} \hat{w}\right\}\right] \\
& \hat{Z}=\frac{1}{|A|}\left[\lambda_{k y} \lambda_{l c} \hat{w}-\lambda_{l y} \lambda_{k y} \sigma_{y} \hat{r}\left(\theta_{k y}+\theta_{l y}\right)+\lambda_{k y} \lambda_{l z} \sigma_{z} \theta_{k z}(\hat{w}-\hat{r})\right. \\
& \left.\quad+\lambda_{l y} \lambda_{k z} \sigma_{z} \theta_{l z}(\hat{w}-\hat{r})+\lambda_{l y} \lambda_{k x} \sigma_{x}\left(\hat{w}_{s}-\hat{r}\right)\right] \\
& \hat{L_{c}}=\frac{1}{|A|}\left[-\lambda_{l y} \lambda_{k y} \sigma_{y} \hat{r}\left(\theta_{k y}+\theta_{l y}\right)-\bar{w}\left(\lambda_{k y} \lambda_{l z}-\lambda_{l y} \lambda_{k z}\right)\right. \\
& \left.\quad+\lambda_{k y} \lambda_{l z} \sigma_{z} \theta_{k z}(\hat{w}-\hat{r})+\lambda_{l y} \lambda_{k z} \sigma_{z} \theta_{l z}(\hat{w}-\hat{r})+\lambda_{l y} \lambda_{k x} \sigma_{x}\left(\hat{w_{s}}-\hat{r}\right)\right] \\
& \text { Here, }|A|=\left|\begin{array}{lll}
\lambda_{k y} & \lambda_{k z} & 0 \\
\lambda_{l y} & \lambda_{l z} & \lambda_{l c} \\
0 & -1 & 1
\end{array}\right|=\lambda_{k y}\left(\lambda_{l c}+\lambda_{l z}\right)-\lambda_{k z} \lambda_{l y}
\end{aligned}
$$

Note that $\left(1-\lambda_{k x}\right)=\left(\lambda_{k y}+\lambda_{k z}\right)$. And if $\mathrm{Y}$ is relatively capital intensive, $\lambda_{k y}>\lambda_{k z}$ or, $\lambda_{l z}>\lambda_{l y}$. Therefore it is most likely that $|A|>0$.

Thus, if capitalists are the worst sufferers among all factors, i.e $\left(\hat{w}_{s}-\hat{r}\right)>0$, $\hat{X}>0 ; \hat{Y}<0 ; \hat{Z}>0$. The underlying economic argument is quite easy to understand. Skilled wage falls at slower rate than $r$. Hence producer economizes on the usage of skilled labor. This induces an increase in $X$. However, it should be noted that $w_{s}$ may even rise under certain condition stated before. In that case our result would be apparent and more robust. Similar kind of argument raises the output of $Z$. This will ensure an unambiguous fall in $Y$ as $X, Y$ and $Z$ shares the same capital. Nevertheless, the value of $L_{C}$ is ambiguous. Since, on the one hand as $\mathrm{Y}$ falls, some unskilled laborers are released and on the other hand as $\mathrm{Z}$ goes up, some unskilled laborers get employed in informal production. Thus, we need to weigh these two countervailing effects to arrive at the eventual effect on $L_{C}$. 


$$
\begin{aligned}
& \hat{L_{C}} \gtrless 0 \text { iff } \\
& \qquad \begin{array}{l}
\left|\lambda_{k y} \lambda_{l z} \sigma_{z} \theta_{k z}(\hat{w}-\hat{r})+\lambda_{l y} \lambda_{k z} \sigma_{z} \theta_{l z}(\hat{w}-\hat{r})+\lambda_{l y} \lambda_{k x} \sigma_{x}\left(\hat{w_{s}}-\hat{r}\right)-\lambda_{l y} \lambda_{k y} \sigma_{y} \hat{r}\left(\theta_{k y}+\theta_{l y}\right)\right| \gtrless \\
\quad\left|\hat{w}\left(\lambda_{k y} \lambda_{l z}-\lambda_{l y} \lambda_{k z}\right)\right|
\end{array}
\end{aligned}
$$

A careful investigation of equation (24) reveals that an increase in informal wage, essentially, dampens the positive effect on $L_{C}$. This is also evident from equation (14). The change in $Y$ and $Z$ are very crucial as both these goods share the same labor with the intermediation/extortion sector. All the effects are simultaneously captured in equation (22). However, it is most likely that $L_{C}$ should increase consequent upon recession. The released capital from $\mathrm{Y}$ is distributed among $\mathrm{X}$ and $\mathrm{Z}$, but the released unskilled labor from $\mathrm{Y}$ has the only productive employment option in $Z$. And subsequently, higher production of $Z$ must call for more intermediators to sort out the problem of negotiation. If $L_{C}$ increases, the size of the intermediation sector $\left(w \cdot L_{C}\right)$ would increase unambiguously as $\hat{w}>0$.

Thus the following proposition is immediate:

Proposition : Consequent upon recession the size of the intermediation sector must expand if $Y$ is relatively capital-intensive.

\section{Concluding Remarks}

In this paper I have constructed a general equilibrium trade model using the hybrid structure of Heckscher-Ohlin and Specific Factor model with a corrupt informal sector to substantiate what could happen to the size of the intermediation sector due to an economic recession. It has been shown that the size will increase if unskilled labor using formal sector is relatively capital intensive than its informal counterpart. However, the aspect of capital immobility between formal and informal sector may induce an interesting dimension to the result that has been derived here. If capital can not move across sectors and $Y$ falls consequent upon recession, some unskilled labor would rush to the informal sector. Thus $w$ would be depressed unlike the basic model. Nevertheless $Z$ must increase as $R$ rises. Therefore the size of the intermediation sector $\left(=\alpha \cdot P_{z} \cdot Z\right)$ must expand. This will in turn guarantee an unconditional rise in $L_{C}$ or the number of intermediators in the economy. 
Received 21 May 2011, Revised 12 July 2011, Accepted 22 July 2011

\section{References}

Bhagwati, J. (1982), Directly Unproductive Profit Seeking (DUP) Activities. Journal of Political Economy, 90(5), pp. 988-1002.

Chaudhuri, S. (2009), Economic Recession and Informal Sector Workers, MPRA paper no.18033. http://mpra.ub.uni-muenchen.de/18033/.

Dijkstra, B. R. (2006), Good and bad equilibria with informal sector, University of Nottingham, School of Economics, Discussion paper 06/01.

Friedman, E., S. Johnson, D. Kaufmann, and P. Zoido-Lobaton. (2000), Dodging the grabbing hand: the determinants of unofficial activity in 69 countries, Journal of Public Economics, 76(3), pp. 459-493.

Gruen, F. and M. Corden. (1970), A tariff that worsens terms of trade, In I. A. McDougall and R. H. Snapes (Eds) Studies in International Economics, Amsterdam: NorthHolland.

Jones, R.W. (1965), The Structure of Simple General Equilibrium Models. Journal of Political Economy, 73(6), pp.557-572.

Jones, R.W. (1971), A three-factor model in theory, trade and history. In Bhagwati, J et al (Eds) Trade, Balance of Payments and Growth, North- Holland, Amsterdam, pp. 321.

Jones, R.W. and S. Marjit. (1992), International trade and endogenous production structure. In Neuefeind, W et al (Eds) Economic Theory and International Trade: Essays in honour of J. Trout Rader, Sringer-Verlag.

Jones, R.W. and S. Marjit. (2009), Competitive trade models and real world features. Economic Theory; 41(1), pp. 163-174.

Mandal, B. and S. Chaudhuri. (2011), Informal Wage, Informal Price and Extortion under Migration and Tariff Reform. Modern Economy, 2(1), pp. 38-43.

Mandal, B. and S. Marjit. (2010), Corruption and Wage Inequality?. International Review of Economics and Finance, 19, pp. 166-172.

Marjit, S. (2009), Firm Heterogeneity, Informal Wage and Good Governance. MPRA paper no. 19168. http://mpra.ub.uni-muenchen.de/19168/.

Marjit, S., S. Kar, and S. Chaudhuri. (2011), Recession in the skilled sector and Implications for informal wage, Research in Economics, 65(3), pp 158-163.

Marjit, S. and S. Kar (2009), A Contemporary Perspective on the Informal Labor Market: Theory, Policy and the Indian experience, Economic and Political Weekly; Vol. XLIV No.14.

Marjit, S and B. Mandal. (2008), Corruption and Trade in General Equilibrium, Leverhulme Centre for GEP, University of Nottingham, RP No. 2008/15.

UNCTAD. (2009) - Global economic crisis: implications for trade and development, Report by UNCTAD Secretariat. 